Article

\title{
The Average Hull Dimension of Negacyclic Codes over Finite Fields
}

\author{
Somphong Jitman ${ }^{1}$ (D) and Ekkasit Sangwisut ${ }^{2, *}$ \\ 1 Department of Mathematics, Faculty of Science, Silpakorn University, Nakhon Pathom 73000, Thailand; \\ sjitman@gmail.com \\ 2 Department of Mathematics and Statistics, Faculty of Science, Thaksin University, \\ Phattalung 93110, Thailand \\ * Correspondence: ekkasit.sangwisut@gmail.com
}

Received: 6 August 2018; Accepted: 17 August 2018; Published: 20 August 2018

\begin{abstract}
Hulls of linear codes have been extensively studied due to their wide applications and links with the efficiency of some algorithms in coding theory. In this paper, the average dimension of the Euclidean hull of negacyclic codes of length $n$ over finite fields $\mathbb{F}_{q}$, denoted by $E(n,-1, q)$, has been investigated. The formula for $E(n,-1, q)$ has been determined. Some upper and lower bounds of $E(n,-1, q)$ have been given as well. Asymptotically, it has been shown that either $E(n,-1, q)$ is zero or it grows the same rate as $n$.
\end{abstract}

Keywords: average hull dimension; negacyclic codes; hulls; self-reciprocal polynomials

MSC: 94B15, 94B05.

\section{Introduction}

In practice, communication systems are not $100 \%$ reliable due to noise or other forms of interference. Coding theory is a branch of Engineering Mathematics that has been introduced and applied to solve this problem since the 1960s. Codes have later been extensively studied and linked with other problems and applications.

In 1990, the (Euclidean) hull of a linear code has been introduced to classify finite projective planes in [1]. It is defined to be the intersection of a linear code and its Euclidean dual. Hulls of linear codes have various applications and play an important role in the efficiency determination of some algorithms in coding theory such as computing permutation equivalence of two linear codes and finding the automorphism group of linear codes (see, for example, [2-6]). Precisely, the efficiency of these computations is limited by the hull size of codes. In [7], the hulls of linear codes have been applied in constructing good entanglement-assisted quantum error correcting codes.

Properties of hulls of codes have been extensively studied. The average dimensions of the Euclidean hull of linear codes and of cyclic codes were given in $[8,9]$, respectively. The dimensions of the hulls of cyclic codes and negacyclic codes were determined in [10]. Later, the complete study of the average dimension of the Hermitian hull of cyclic and constacyclic codes was given in [11,12]. It is of natural interest to study the average dimension of the Euclidean hull of constacyclic codes. In [13], it has been shown that the Euclidean dual of $\lambda$-constacyclic code is again $\lambda$-constacyclic if and only if $\lambda= \pm 1$. Therefore, the average dimension of the Euclidean hull of negacyclic codes $(\lambda=-1)$ is the only remaining case.

In this paper, we focus on the average dimension of the Euclidean hull of negacyclic codes of length $n$ over finite fields $\mathbb{F}_{q}$ as well as its lower and upper bounds. The paper is organized as follows. Basic properties of codes and polynomials over finite fields are recalled in Section 2. In Section 3, 
the expression for $E(n,-1, q)$, the formula for the average dimension of neagcyclic codes, is given together with some upper bounds. In Section 4, upper and lower bounds on $E(n,-1, q)$ are derived. The summary and remarks are given in Section 5.

\section{Preliminaries}

Let $p$ be a prime and let $q$ be a $p$-power. Denote by $\mathbb{F}_{q}$ the finite field of order $q$ and characteristic $p$. For given positive integers $k \leq n$, a linear code of length $n$ and dimension $k$ over $\mathbb{F}_{q}$ is a $k$-dimensional subspace of the $\mathbb{F}_{q}$-vector space $\mathbb{F}_{q}^{n}$. The Euclidean dual of a linear code $C$ is defined to be

$$
C^{\perp}=\left\{\left(u_{0}, u_{1} \ldots, u_{n-1}\right) \in \mathbb{F}_{q}^{n}: \sum_{i=0}^{n-1} u_{i} c_{i}=0 \text { for all }\left(c_{0}, c_{1} \ldots, c_{n-1}\right) \in C\right\} .
$$

The Euclidean hull of a linear code $C$ is defined to be

$$
\operatorname{Hull}(C)=C \cap C^{\perp}
$$

A linear code of length $n$ over $\mathbb{F}_{q}$ is said to be negacyclic if $\left(-c_{n-1}, c_{0}, \ldots, c_{n-2}\right) \in C$ for all $\left(c_{0}, c_{1}, \ldots, c_{n-1}\right) \in C$.

Let $\mathcal{C}(n,-1, q)$ denote the set of all neagcyclic codes of length $n$ over $\mathbb{F}_{q}$. The average dimension of the hull of negacyclic codes of length $n$ over $\mathbb{F}_{q}$ is defined to be

$$
E(n,-1, q):=\sum_{C \in \mathcal{C}(n,-1, q)} \frac{\operatorname{dim} \operatorname{Hull}(C)}{|\mathcal{C}(n,-1, q)|}
$$

Every non-zero negacyclic code $C$ of length $n$ over $\mathbb{F}_{q}$ can be viewed as an ideal of the principal ideal ring $\mathbb{F}_{q}[x] /\left\langle x^{n}+1\right\rangle$ generated by a monic divisor $g(x)$ of $x^{n}+1$ (see [10]). In this case, $g(x)$ is called the generator polynomial for $C$ and $\operatorname{dim} C=n-\operatorname{deg}(g(x))$.

For a polynomial $f(x)=a_{0}+a_{1} x+\cdots+a_{k} x^{k} \in \mathbb{F}_{q}[x]$ of degree $k$ and $a_{0} \neq 0$, the reciprocal polynomial of $f(x)$ is defined to be $f^{*}(x):=f(0)^{-1} x^{\operatorname{deg} f(x)} f\left(\frac{1}{x}\right)$. It is not difficult to see that $\left(f^{*}\right)^{*}(x)=f(x)$. Then, we have two types of polynomials. A polynomial $f(x)$ is called self-reciprocal if $f(x)=f^{*}(x)$. Otherwise, $f(x)$ and $f^{*}(x)$ are called a reciprocal polynomial pair.

Let $C$ be a negacyclic code of length $n$ over $\mathbb{F}_{q}$ with the generator polynomial $g(x)$ and let $h(x)=\frac{x^{n}+1}{g(x)}$. Then, $h^{*}(x)$ is a monic divisor of $x^{n}+1$ and it is the generator polynomial of $C^{\perp}$ by Lemma 2.1 of [13]. Therefore, Hull(C) is generated by the polynomial $\operatorname{lcm}\left(g(x), h^{*}(x)\right)$ (see Theorem 1 of [10]).

Recall that the characteristic of $\mathbb{F}_{q}$ is $p$. Then, a positive integer $n$ can be written in the form of $n=\bar{n} p^{v}$, where $p \nmid \bar{n}$ and $v \geq 0$. Using arguments similar to those in Section 4 of [10], up to permutation, there exist nonnegative integers $s$ and $t$ such that

$$
x^{n}+1=\left(x^{\bar{n}}+1\right)^{p^{v}}=\prod_{i=1}^{\mathbf{s}} g_{i}(x)^{p^{v}} \prod_{j=1}^{\mathrm{t}} f_{j}(x)^{p^{v}} f_{j}^{*}(x)^{p^{v}}
$$

where $f_{j}(x)$ and $f_{j}^{*}(x)$ are a reciprocal polynomial pair and $g_{i}(x)$ is a monic irreducible self-reciprocal polynomial for all $1 \leq i \leq \mathrm{s}$ and $1 \leq j \leq \mathrm{t}$.

For a given negacyclic code $C$ of length $n$ over $\mathbb{F}_{q}$, based on the factorization in (1), the generator polynomial of $C$ can be viewed of the form

$$
g(x)=\prod_{i=1}^{\mathrm{s}} g_{i}(x)^{u_{i}} \prod_{j=1}^{\mathrm{t}} f_{j}(x)^{z_{j}}\left(f_{j}^{*}(x)\right)^{w_{j}}
$$


where $0 \leq u_{i}, z_{j}, w_{j} \leq p^{v}$. It follows that the generator polynomial of $C^{\perp}$ is

$$
h^{*}(x)=\prod_{i=1}^{\mathrm{s}} g_{i}(x)^{p^{v}-u_{i}} \prod_{j=1}^{\mathrm{t}} f_{j}(x)^{p^{v}-w_{j}}\left(f_{j}^{*}(x)\right)^{p^{v}-z_{j}},
$$

and hence the generator polynomial of $\operatorname{Hull}(C)$ is

$$
\operatorname{lcm}\left(g(x), h^{*}(x)\right)=\prod_{i=1}^{\mathbf{s}} g_{i}(x)^{\max \left\{u_{i}, p^{v}-u_{i}\right\}} \prod_{j=1}^{\mathrm{t}} f_{j}(x)^{\max \left\{z_{j}, p^{v}-w_{j}\right\}}\left(f_{j}^{*}(x)\right)^{\max \left\{w_{j}, p^{v}-z_{j}\right\}} .
$$

Since 1 and -1 are identical when the characteristic of $\mathbb{F}_{q}$ is even, in the rest of this paper, we assume that the characteristic $p$ of $\mathbb{F}_{q}$ is odd.

\section{The Average Dimension $E(n,-1, q)$}

In this section, we focus on an explicit expression for the formula of the average dimension of the Euclidean hull of negacyclic codes of length $n$ over $\mathbb{F}_{q}$. Employing techniques similar to those for the cyclic case in [9], slightly different results for the negacyclic case can be deduced.

Assume that $x^{\bar{n}}+1$ has the factorization in the form of Equation (1) and let $B_{\bar{n},-1, q}=\sum_{i=1}^{\mathbf{s}} \operatorname{deg} g_{i}(x)$. The expectation $E(\cdot)$ in Lemma 1 can be obtained using arguments similar to those in the proof of Proposition 22 of [9] .

Lemma 1. Let $p$ be an odd prime and let $v$ be a nonnegative integer. Let $0 \leq u, z, w \leq p^{v}$. Then, the following statements hold:

1. $E\left(\max \left\{u, p^{v}-u\right\}\right)=\frac{3 p^{v}+1}{4}$.

2. $E\left(\max \left\{z, p^{v}-w\right\}\right)=\frac{\left.p^{v} 4 p^{v}+5\right)}{6\left(p^{v}+1\right)}$.

The average dimension of the Euclidean hull of neagcyclic codes of length $n$ over $\mathbb{F}_{q}$ can be determined as follows.

Theorem 1. Let $\mathbb{F}_{q}$ be a finite field of order $q$ and odd characteristic $p$ and let $n$ be a positive integer such that $n=\bar{n} p^{v}, p \nmid \bar{n}$ and $v \geq 0$. Then, the average dimension of the Euclidean hull of negacyclic codes of length $n$ over $\mathbb{F}_{q}$ is

$$
E(n,-1, q)=n\left(\frac{2 p^{v}+1}{6\left(p^{v}+1\right)}\right)-B_{\bar{n},-1, q}\left(\frac{p^{2 v}+2 p^{v}+3}{12\left(p^{v}+1\right)}\right) .
$$

Proof. By Lemma 1, Equation (2), and arguments similar to those in the proof of Theorem 3.2 of [11], it can be deduced that

$$
\begin{aligned}
E(n,-1, q) & =n\left(\frac{1}{3}-\frac{1}{6\left(p^{v}+1\right)}\right)-B_{\bar{n},-1, q}\left(\frac{p^{v}+1}{12}+\frac{2}{12\left(p^{v}+1\right)}\right) \\
& =n\left(\frac{2 p^{v}+1}{6\left(p^{v}+1\right)}\right)-B_{\bar{n},-1, q}\left(\frac{p^{2 v}+2 p^{v}+3}{12\left(p^{v}+1\right)}\right) .
\end{aligned}
$$

This completes the proof.

The next corollary is a direct consequence of Theorem 1.

Corollary 1. Assume the notations as in Theorem 1. Then, the following statements hold:

1. $E(n,-1, q)<\frac{n}{3}$.

2. $E(\bar{n},-1, q)=\frac{\frac{3}{n}-B_{\bar{n},-1, q}}{4}$.

3. $E(\bar{n},-1, q)<\frac{\bar{n}}{4}$. 


\section{Properties of $B_{\bar{n},-1, q}$ and Bounds on $E(n,-1, q)$}

In this section, some number theoretical tools are constructed and applied to study properties of $B_{\bar{n},-1, q}$. As a consequence, lower and upper bounds for $E(n,-1, q)$ can be derived using $B_{\bar{n},-1, q}$.

For an odd prime power $q$, let $N_{q}:=\left\{\ell \geq 1: \ell\right.$ divides $\left.q^{i}+1\right\}$. For coprime positive integers $i$ and $j$, denote by $\operatorname{ord}_{j}(i)$ the multiplicative order of $i$ modulo $j$. An element in $N_{q}$ has the following properties.

Lemma 2. Let $q$ be an odd prime power. If $\ell \in N_{q}$ and $\ell>2$, then $\operatorname{ord}_{\ell}(q)$ is even.

Proof. Since $\ell \in N_{q}$, there exists the smallest positive integer $k$ such that $\ell \mid\left(q^{k}+1\right)$. It follows that $\ell \mid\left(q^{2 k}-1\right)$. Then, $\operatorname{ord}_{\ell}(q) \mid 2 k$. Since $\operatorname{ord}_{\ell}(q) \nmid k$, $\operatorname{ord}_{\ell}(q)$ is even.

Next, we introduce a partition for the set $N_{q}$. For each integer $\alpha \geq 0$, let

$$
P_{q, \alpha}:=\left\{\ell \in N_{q}: 2^{\alpha}|| \operatorname{ord}_{\ell}(q)\right\},
$$

where $2^{\alpha}|| k$ is used if $\alpha$ is the integer such that $2^{\alpha} \mid k$ and $2^{\alpha+1} \nmid k$. Then, we have $N_{q}=P_{q, 0} \cup P_{q, 1} \cup$ $P_{q, 2} \cdots$.

Theorem 2 (Theorem 4 of [9]). Let $q$ be an odd prime power and let $\ell$ be a positive integer. Then, the following statements hold:

1. Let $\ell$ be an odd integer. If $\ell>1$ is such that $\ell=\prod_{i=1}^{k} p_{i}^{e_{i}}$ the prime factorization of $\ell$. Then, $\ell \in N_{q}$ if and only if there exists $\alpha>0$ such that $p_{i} \in P_{q, \alpha}$ for all $i$. In this case, we have $\ell \in P_{q, \alpha}$.

2. Let $\beta \geq 1$ be an integer. Then $2^{\beta} \in N_{q}$ if and only if $2^{\beta}$ divides $q+1$. Moreover, if $2^{\beta} \in N_{q}, \beta \geq 2$, then $2^{\beta} \in P_{q, 1}$.

3. Let $q$ and $\ell$ be odd. Then, $2 \ell \in N_{q}$ if and only if $\ell \in N_{q}$. In this case, $\ell$ and $2 \ell$ belong to the same set $P_{q, \alpha}$.

4. Let $\ell=2^{\beta} \bar{\ell}$ where $\bar{\ell}$ is odd and $\beta \geq 2$. Then, $\ell \in N_{q}$ if and only if $2^{\beta} \in N_{q}$ and $\bar{\ell} \in P_{q, 1}$. In this case, we have $\ell \in P_{q, 1}$.

The characterization of elements in $P_{q, \alpha}$ are given in the following corollary.

Corollary 2. Let $\gamma \geq 1$ be an integer such that $2^{\gamma} \mid(q+1)$. Let $\ell$ be a positive integer relatively prime to $q$ and let $2^{\beta} \| \ell$. Then, the following statements hold:

1. $P_{q, 0}=\{1,2\}$.

2. $\ell \in P_{q, 1}$ if and only if either $\ell$ has an odd prime divisor, each odd prime divisor of $\ell$ belongs to $P_{q, 1}$ and $0 \leq \beta \leq \gamma$, or $\ell=2^{\beta}$ and $2 \leq \beta \leq \gamma$.

3. Let $\alpha \geq 2$. Then, $\ell \in P_{q, \alpha}$ if and only if $\ell$ has an odd prime divisor, each odd prime divisor of $\ell$ belongs to $P_{q, \alpha}$ and $0 \leq \beta \leq 1$.

Lemma 3. Let $\alpha \geq 1$ an integer and let $\ell$ be a positive integer. If $\ell \in P_{q, \alpha}$, then $\ell \geq 2^{\alpha}+1$.

Proof. By Corollary 2, we have $\ell \geq 3$. Since $\ell \in P_{q, \alpha}$, it follows that $2^{\alpha} \| \operatorname{ord}_{\ell}(q)$. By Little Fermat's Theorem, we have $\operatorname{ord}_{\ell}(q) \mid \phi(\ell)$. Then, $2^{\alpha} \mid \phi(\ell)$. Hence, $2^{\alpha} \leq \phi(\ell) \leq \ell-1$.

Let $\Omega:=\{j \in \mathbb{N}: j \mid 2 \bar{n}$ and $2 \nmid \bar{n}\}$. Next, we give the expression of $B_{\bar{n},-1, q}$.

Lemma 4. Assume that $x^{\bar{n}}+1$ is factorized as in Equation (1). Then,

$$
B_{\bar{n},-1, q}=\sum_{j \in \Omega \cap N_{q}} \phi(j)
$$

where $\phi$ is the Euler's totient function. 
Proof. By Equation (1), we have

$$
x^{\bar{n}}+1=\prod_{i=1}^{\mathrm{s}} g_{i}(x) \prod_{j=1}^{\mathrm{t}} f_{j}(x) f_{j}^{*}(x)
$$

From Equation (29) of [10], $x^{\bar{n}}+1$ can be factored as

$$
x^{\bar{n}}+1=\prod_{j \in \Omega \cap N_{q}} \prod_{i=1}^{\gamma(j)} g_{i j}(x) \prod_{j \in \Omega \backslash N_{q}} \prod_{i=1}^{\beta(j)} f_{i j}(x) f_{i j}^{*}(x),
$$

where $\gamma(j)=\frac{\phi(j)}{\operatorname{ord}_{j}(q)}, \beta(j)=\frac{\phi(j)}{2 \operatorname{ord}_{j}(q)}, f_{i j}(x)$ and $f_{i j}^{*}(x)$ are a monic irreducible-reciprocal polynomial pair of degree $\operatorname{ord}_{j}(q)$, and $g_{i j}(x)$ is a monic irreducible self-reciprocal polynomial of degree $\operatorname{ord}_{j}(q)$.

Altogether, it can be concluded that

$$
\prod_{i=1}^{\mathrm{s}} g_{i}(x)=\prod_{j \in \Omega \cap N_{q}} \prod_{i=1}^{\gamma(j)} g_{i j}(x) .
$$

Hence,

$$
B_{\bar{n},-1, q}=\sum_{i=1}^{\mathbf{s}} \operatorname{deg}\left(g_{i}(x)\right)=\sum_{j \in \Omega \cap N_{q}} \gamma(j) \operatorname{deg}\left(g_{i j}(x)\right)=\sum_{j \in \Omega \cap N_{q}} \frac{\phi(j)}{\operatorname{ord}_{j}(q)} \cdot \operatorname{ord}_{j}(q)=\sum_{j \in \Omega \cap N_{q}} \phi(j)
$$

as desired.

Remark 1. From Lemma 4, we have the following facts. The set $\Omega \cap N_{q}$ can be empty. For convenience, the empty summation will be regarded as 0 . In this case, $B_{\bar{n},-1, q}=\sum_{j \in \Omega \cap N_{q}} \phi(j)=\sum_{j \in \varnothing} \phi(j)=0$. For example, $B_{4,-1,3}=0$ since $\Omega \cap N_{3}=\varnothing$.

The expression of the set $\Omega$ can be simplified using the definition of $\bar{n}$ as follows.

Lemma 5. Write $\bar{n}=2^{\beta} n^{\prime}$, where $n^{\prime}$ is an odd integer and $\beta$ is a non-negative integer. Then, $\Omega=$ $\left\{2^{\beta+1} k: k \in \mathbb{N}\right.$ and $\left.k \mid n^{\prime}\right\}$.

Proof. Let $\bar{n}=2^{\beta} n^{\prime}$. Then, we have $\Omega=\{j \in \mathbb{N}: j \mid 2 \bar{n}$ and $2 \nmid \bar{n}\}=\left\{j \in \mathbb{N}: j \mid 2^{\beta+1} n^{\prime}\right.$ and $\left.j \nmid 2^{\beta} n^{\prime}\right\}$. Hence, $2^{\beta+1} \mid j$ for all $j \in \Omega$, which implies $\Omega=\left\{2^{\beta+1} k: k \in \mathbb{N}\right.$ and $\left.k \mid n^{\prime}\right\}$.

The following result is a consequence of Lemma 5 and Theorem 2.

Proposition 1. Let $\gamma \geq 1$ be the integer such that $2^{\gamma} \|(q+1)$. Then, the following statements hold:

1. $\Omega \cap N_{q}=\varnothing$ if and only if $\beta+1>\gamma$.

2. $\Omega \cap N_{q}=\Omega$ if and only if $\beta+1 \leq \gamma$ and $2 \bar{n} \in N_{q}$.

3. $\varnothing \subsetneq \Omega \cap N_{q} \subsetneq \Omega$ if and only if $\beta+1 \leq \gamma$ and $2 \bar{n} \notin N_{q}$.

Proof. To prove (i), assume that $\beta+1 \leq \gamma$. Then, by Theorem 2, it can be concluded that $2^{\beta+1} \in N_{q}$. Hence, $\Omega \cap N_{q} \neq \varnothing$.

Conversely, assume that $\beta+1>\gamma \geq 1$. Then, $\beta+1 \geq 2$. Let $j \in \Omega$. By Lemma $5, j=2^{\beta+1} k$ for some $k \mid n^{\prime}$. Therefore, $j \notin N_{q}$ by Theorem 2, which implies $\Omega \cap N_{q}=\varnothing$.

To prove (ii), assume that $\Omega \cap N_{q}=\Omega$. Then, $\Omega \subseteq N_{q}$. Since $2 \bar{n} \in \Omega$, we have $2 \bar{n} \in N_{q}$. Hence, $2^{\beta+1} n^{\prime}=2 \bar{n} \in N_{q}$ which implies $\beta+1 \leq \gamma$ by Theorem 2 .

Conversely, assume that $\beta+1 \leq \gamma$ and $2 \bar{n} \in N_{q}$. Let $j \in \Omega$. Then, $j \mid 2 \bar{n}$. Since $2 \bar{n} \in N_{q}, j \in N_{q}$, $\Omega \subseteq N_{q}$. 
Statement (iii) follows immediately from (i) and (ii).

By Proposition 1, we have the following corollary.

Corollary 3. Let $\gamma \geq 1$ be the integer such that $2^{\gamma} \|(q+1)$. Then, the following statements hold:

1. $B_{\bar{n},-1, q}=0$ if and only if $\beta+1>\gamma$.

2. $B_{\bar{n},-1, q}=\bar{n}$ if and only if $\beta+1 \leq \gamma$ and $2 \bar{n} \in N_{q}$.

3. $0<B_{\bar{n},-1, q}<\bar{n}$ if and only if $\beta+1 \leq \gamma$ and $2 \bar{n} \notin N_{q}$.

Proof. By Proposition $1, \beta+1>\gamma$ if and only if $\Omega \cap N_{q}=\varnothing$. Equivalently,

$$
B_{\bar{n},-1, q}=\sum_{j \in \Omega \cap N_{q}} \phi(j)=\sum_{j \in \varnothing} \phi(j)=0 .
$$

This proves (i).

By Proposition $1, \beta+1 \leq \gamma$ and $2 \bar{n} \in N_{q}$ if and only if $\Omega \cap N_{q}=\Omega$. Equivalently,

$$
B_{\bar{n},-1, q}=\sum_{j \in \Omega \cap N_{q}} \phi(j)=\sum_{j \in \Omega} \phi(j)=\sum_{k \mid n^{\prime}} \phi\left(2^{\beta+1} k\right)=2^{\beta} \sum_{k \mid n^{\prime}} \phi(k)=2^{\beta} n^{\prime}=\bar{n} .
$$

Statement (iii) can be deduced directly from (i) and (ii).

Corollary 4. Assume the notations as above. Then, the following statements hold:

1. $E(n,-1, q)=n\left(\frac{1}{3}-\frac{1}{6\left(p^{v}+1\right)}\right)$ if and only if $\beta+1>\gamma$.

2. If $\beta+1>\gamma$, then $\frac{n}{4} \leq E(n,-1, q)<\frac{n}{3}$.

Proof. The first statement can be deduced directly from Theorem 1 and Corollary 3. The second statement follows from Corollary 1 and the fact that $\frac{1}{6\left(p^{v}+1\right)}$ reaches its maximum value $\frac{1}{12}$ when $v=0$.

Next, we focus on the case where $\beta+1 \leq \gamma$. Let $\ell$ be a positive integer relatively prime to $q$. Let $\ell=2^{\beta} p_{1}^{e_{1}} \ldots p_{k}^{e_{k}}$ be the prime factorization of $\ell$, where $\beta \geq 0, k \geq 0, p_{1}, p_{2}, \ldots, p_{k}$ are distinct odd primes, and $e_{i} \geq 1$ for all $i=1,2, \ldots, k$. Partition the index set $\{1, \ldots, k\}$ into $K^{\prime}, K_{1}, K_{2}, \ldots$ as follows:

1. $i \in K^{\prime}$ if $p_{i} \notin P_{q, \alpha}$ for all $\alpha \geq 1$,

2. $i \in K_{\alpha}$ if $p_{i} \in P_{q, \alpha}$.

Let $d^{\prime}=\prod_{i \in K^{\prime}} p_{i}^{e_{i}}$ and $d_{\alpha}=\prod_{i \in K_{\alpha}} p_{i}^{e_{i}}$ for all $1 \leq \alpha \leq k$. For convenience, the empty product will be referred to as 1 . Therefore, we have $\ell=2^{\beta} d^{\prime} d_{1} d_{2} \ldots$ which is called the $N_{q}$-factorization of $\ell$, where $d_{i}=1$ for all but finitely many integers $i$. By Theorem 2 , we have $d_{\alpha} \in P_{q, \alpha}$. The characterization of $\ell \notin N_{q}$ is given in the following lemma.

Lemma 6 (Lemma 9 of [9]). Let $\gamma \geq 1$ be the integer such that $2^{\gamma} \|(q+1)$. Let $\ell \geq 2$ be such that $\operatorname{gcd}(\ell, q)=1$ and let $\ell=2^{\beta} d^{\prime} d_{1} d_{2} \ldots$ be the $N_{q}$-factorization of $\ell$. If $\ell \notin N_{q}$, then at least one of the following conditions is valid:

1. $\beta>\gamma$.

2. $d^{\prime}>1$.

3. $\beta \geq 2$ and $d_{\alpha}>1$ for an integer $\alpha \geq 2$.

4. $\quad d_{\alpha_{1}}>1$ and $d_{\alpha_{2}}>1$ for two distinct $\alpha_{1} \geq 1$ and $\alpha_{2} \geq 1$.

The following proposition provides a simplified expression of $B_{\bar{n},-1, q}$. 
Proposition 2. Let $\bar{n}=2^{\beta} d^{\prime} d_{1} d_{2} \ldots$ be an $N_{q}$-factorization of $\bar{n}=2^{\beta} n^{\prime}$. If $\beta+1 \leq \gamma$ and $2 \bar{n} \notin N_{q}$, then

$$
B_{\bar{n},-1, q}= \begin{cases}d_{1}+\sum_{\alpha \geq 2}\left(d_{\alpha}-1\right), & \text { if } \beta=0, \\ 2^{\beta} d_{1}, & \text { if } \beta \neq 0 .\end{cases}
$$

Proof. We distinguish the proof into two cases where $\beta=0$ and $\beta \neq 0$.

Case $1 \beta=0$. We have $\bar{n}=d^{\prime} d_{1} d_{2} \ldots=n^{\prime}$. By Lemma 5 and Theorem 2 , we have

$$
\begin{aligned}
B_{\bar{n},-1, q} & =\sum_{j \in \Omega \cap N_{q}} \phi(j)=\sum_{k \mid \bar{n}, 2 k \in N_{q}} \phi(2 k)=\phi(2)+\sum_{\alpha \geq 1} \sum_{k \mid d_{\alpha}, k \neq 1} \phi(2 k) \\
& =1+\sum_{\alpha \geq 1} \sum_{k \mid d_{\alpha}, k \neq 1} \phi(k)=1+\sum_{\alpha \geq 1}\left(d_{\alpha}-1\right)=d_{1}+\sum_{\alpha \geq 2}\left(d_{\alpha}-1\right) .
\end{aligned}
$$

Case $2 \beta \neq 0$. We have $\beta+1 \geq 2$ and $\bar{n}=2^{\beta} d^{\prime} d_{1} d_{2} \ldots=2^{\beta} n^{\prime}$. By Corollary 2 , we have $2^{\beta+1} k \in N_{q}$. Since $k \mid n^{\prime}$ if and only if $k \mid d_{1}$, it follows that

$$
B_{\bar{n},-1, q}=\sum_{j \in \Omega \cap N_{q}} \phi(j)=\sum_{k \mid n^{\prime}, 2^{\beta+1} k \in N_{q}} \phi\left(2^{\beta+1} k\right)=\sum_{k \mid d_{1}} \phi\left(2^{\beta+1} k\right)=2^{\beta} \sum_{k \mid d_{1}} \phi(k)=2^{\beta} d_{1} .
$$

The results follow.

Theorem 3. Let $q$ be an odd prime power and $2^{\gamma} \|(q+1)$. Let $n=\bar{n} p^{v}$, where $p \nmid \bar{n}$ and $v \geq 0$. Let $\bar{n}=2^{\beta} n^{\prime}$, where $2 \nmid n^{\prime}$. Then, the following statements hold:

1. $E(n,-1, q)=0$ if and only if $\beta+1 \leq \gamma, v=0$, and $2 \bar{n} \in N_{q}$.

2. If $\beta+1 \leq \gamma, v>0$, and $2 \bar{n} \in N_{q}$, then $\frac{n}{6} \leq E(n,-1, q)<\frac{n}{4}$.

3. If $\beta+1 \leq \gamma$ and $2 \bar{n} \notin N_{q}$, then $\frac{n}{12} \leq E(n,-1, q)<\frac{n}{3}$.

Proof. By Equation (3), $E(n,-1, q)=0$ if and only if

$$
\frac{B_{\bar{n},-1, q}}{\bar{n}}=\frac{4 p^{2 v}+2 p^{v}}{p^{2 v}+2 p^{v}+3}
$$

By Corollary 3 , it is not difficult to see that $\frac{B_{\bar{n},-1, q}}{\bar{n}} \leq 1$ and $\frac{B_{\bar{n},-1, q}}{\bar{n}}=1$ if and only if $\beta+1 \leq \gamma$ and $2 \bar{n} \in N_{q}$. On the other hand, we have $\frac{4 p^{2 v}+2 p^{v}}{p^{2 v}+2 p^{v}+3} \geq 1$ and $\frac{4 p^{2 v}+2 p^{v}}{p^{2 v}+2 p^{v}+3}=1$ if and only if $p^{v}=1$. Therefore, $\frac{B_{\bar{n},-1, q}}{\bar{n}}=\frac{4 p^{2 v}+2 p^{v}}{p^{2 v}+2 p^{v}+3}$ if and only if $\beta+1 \leq \gamma, 2 \bar{n} \in N_{q}$ and $p^{v}=1$. This proves (i).

To prove (ii), assume that $\beta+1 \leq \gamma, v>0$, and $2 \bar{n} \in N_{q}$. By Corollary 3 , we have $B_{\bar{n},-1, q}=\bar{n}$. By Equation (3), it follows that

$$
E(n,-1, q)=n\left(\frac{2 p^{v}+1}{6\left(p^{v}+1\right)}\right)-\bar{n}\left(\frac{p^{2 v}+2 p^{v}+3}{12\left(p^{v}+1\right)}\right)=\frac{\bar{n}}{12\left(p^{v}+1\right)}\left(3 p^{2 v}-3\right)=\frac{n}{4}\left(1-\frac{1}{p^{v}}\right) .
$$

It is not difficult to see that $E(n,-1, q)<\frac{n}{4}$. Since $v>0$, it follows that $p^{v} \geq 3$. Hence, the minimum value of $1-\frac{1}{p^{v}}$ is $\frac{2}{3}$. Therefore, we have $\frac{n}{6} \leq E(n,-1, q)<\frac{n}{4}$.

To prove (iii), assume that $\beta+1 \leq \gamma$ and $2 \bar{n} \notin N_{q}$.

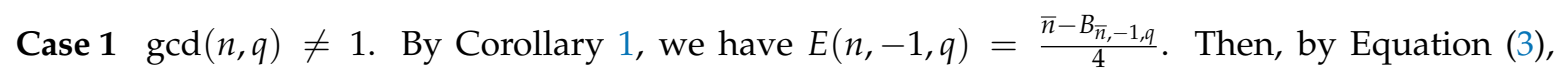
$E(n,-1, q)$ can be expressed as

$$
\frac{E(n,-1, q)}{n}=\frac{1}{4}-\frac{1}{4 p^{v}}+\frac{E(\bar{n},-1, q)}{n}\left(\frac{p^{2 v}+2 p^{v}+3}{3\left(p^{v}+1\right)}\right) \geq \frac{1}{4}-\frac{1}{4 p^{v}}
$$


It is not difficult to see that $\frac{E(n,-1, q)}{n} \geq \frac{1}{6}$ for all $p^{v} \geq 3$. Hence, $E(n,-1, q) \geq \frac{n}{6}$.

Case $2 \operatorname{gcd}(n, q)=1$. Let $\bar{n}=2^{\beta} d^{\prime} d_{1} d_{2} \ldots$ be an $N_{q}$-factorization of $\bar{n}$ and

$$
\bar{n}=2^{\beta} d^{\prime} d_{1} d_{2} \ldots=2^{\beta} d^{\prime} d_{\alpha_{1}} d_{\alpha_{2}} \ldots d_{\alpha_{j}}
$$

where $d_{\alpha_{i}}>1$ for all $1 \leq i \leq j$ and $\alpha_{1}<\alpha_{2}<\cdots<\alpha_{j}$. Note that if $d_{\alpha_{i}}$ and $d^{\prime}$ are greater than 1 , then they are greater than or equal to 3 .

Case 2.1 $\beta=0$. We have $\bar{n}=d^{\prime} d_{\alpha_{1}} d_{\alpha_{2}} \ldots d_{\alpha_{j}}$. It is easy to verify that

$$
\frac{B_{\bar{n},-1, q}}{n}=\frac{d_{1}+\sum_{\alpha \geq 2}\left(d_{\alpha}-1\right)}{d^{\prime} d_{1} d_{2} \ldots}=\frac{1-j+\sum_{i=1}^{j} d_{\alpha_{j}}}{d^{\prime} d_{\alpha_{1}} d_{\alpha_{2}} \ldots d_{\alpha_{j}}} .
$$

By Lemma 6, we have the following six subcases:

Case 2.1.1 $d^{\prime}=1$ and $j=0$. Then, $\bar{n}=1$ and $2 \bar{n}=2 \in N_{q}$, a contradiction.

Case 2.1.2 $d^{\prime}=1$ and $j=1$. Thus, $\bar{n}=d_{\alpha_{1}}, 2 \bar{n}=2 d_{\alpha_{1}} \in N_{q}$, a contradiction.

Case 2.1.3 $d^{\prime}>1$ and $j=0$. We have $\bar{n}=d^{\prime}$. Thus, $\frac{B_{\bar{n},-1, q}}{n}=\frac{1}{d^{\prime}} \leq \frac{1}{3}$.

Case 2.1.4 $d^{\prime}>1$ and $j=1$. Thus, $\bar{n}=d^{\prime} d_{\alpha_{1}}$. Therefore, $\frac{B_{\bar{n},-1, q}}{n}=\frac{d_{\alpha_{1}}}{d^{\prime} d_{\alpha_{1}}}=\frac{1}{d^{\prime}} \leq \frac{1}{3}$.

Case 2.1.5 $j=2$. Hence, $\bar{n}=d^{\prime} d_{\alpha_{1}} d_{\alpha_{2}}$. Without loss of generality, we may assume that $d_{\alpha_{2}} \leq d_{\alpha_{1}}$. We have

$$
\frac{B_{\bar{n},-1, q}}{n}=\frac{-1+d_{\alpha_{1}}+d_{\alpha_{2}}}{d^{\prime} d_{\alpha_{1}} d_{\alpha_{2}}} \leq \frac{2 d_{\alpha_{1}}}{d^{\prime} d_{\alpha_{1}} d_{\alpha_{2}}} \leq \frac{2}{d^{\prime} d_{\alpha_{2}}} \leq \frac{2}{d_{\alpha_{2}}} \leq \frac{2}{3} .
$$

Case 2.1.6 $j \geq 3$. Then, $\bar{n}=d^{\prime} d_{\alpha_{1}} d_{\alpha_{2}} \ldots d_{\alpha_{j}}$. Let $d_{\alpha_{r}}=\max _{1 \leq i \leq j} d_{\alpha_{i}}$.

$$
\frac{B_{\bar{n},-1, q}}{n}=\frac{1-j+\sum_{i=1}^{j} d_{\alpha_{i}}}{d^{\prime} d_{\alpha_{1}} \ldots d_{\alpha_{j}}} \leq \frac{\sum_{i=1}^{j} d_{\alpha_{i}}}{d^{\prime} d_{\alpha_{1}} \ldots d_{\alpha_{j}}} \leq \frac{j d_{\alpha_{r}}}{d^{\prime} d_{\alpha_{1}} \ldots d_{\alpha_{j}}}=\frac{j}{d^{\prime} \prod_{1 \leq i \leq j, i \neq r} d_{\alpha_{i}}} \leq \frac{j}{\prod_{1 \leq i \leq j, i \neq r} d_{\alpha_{i}}} .
$$

Let $s$ be an index such that $j-1 \leq s \leq j$ and $s \neq r$. Then, $j<2^{j-1} \leq 2^{s} \leq 2^{\alpha_{s}}$. Since $d_{\alpha_{s}} \in P_{q, \alpha_{s}}$, we have $d_{\alpha_{s}} \geq 2^{\alpha_{s}}+1$ by Lemma 3. Hence, $j<2^{\alpha_{s}}<d_{\alpha_{s}}$. Therefore,

$$
\frac{B_{\bar{n},-1, q}}{n} \leq \frac{j}{\prod_{1 \leq i \leq j, i \neq r} d_{\alpha_{i}}} \leq \frac{d_{\alpha_{s}}}{\prod_{1 \leq i \leq j, i \neq r} d_{\alpha_{i}}}=\frac{1}{\prod_{1 \leq i \leq j, i \neq r, i \neq s} d_{\alpha_{i}}} \leq \frac{1}{3} .
$$

Case 2.2 $\beta \neq 0$. Then, $\bar{n}=2^{\beta} d^{\prime} d_{1} d_{2} \ldots=2^{\beta} d^{\prime} d_{\alpha_{1}} d_{\alpha_{2}} \ldots d_{\alpha_{j}}$. It follows that

$$
\frac{B_{\bar{n},-1, q}}{n}=\frac{2^{\beta} d_{1}}{2^{\beta} d^{\prime} d_{1} d_{2} \ldots}=\frac{1}{d^{\prime} d_{2} d_{3} \ldots} .
$$

By Lemma 6, we have the following six subcases.

Case 2.2.1 $d^{\prime}=1$ and $j=0$. Then, $\bar{n}=2^{\beta}$ and $2 \bar{n}=2^{\beta+1} \in N_{q}$, a contradiction.

Case 2.2.2 $d^{\prime}=1$ and $j=1$. Note that $\beta+1 \geq 2$. If $\alpha_{1}=1$, then $\bar{n}=2^{\beta} d_{1}$ and $2 \bar{n}=2^{\beta+1} d_{1} \in N_{q}$, a contradiction. Otherwise, $\alpha_{1} \neq 1$. Then, $\bar{n}=2^{\beta} d_{\alpha_{1}}$ and $2 \bar{n}=2^{\beta+1} d_{\alpha_{1}} \notin N_{q}$. Hence,

$$
\frac{B_{\bar{n},-1, q}}{n}=\frac{1}{d_{\alpha_{1}}} \leq \frac{1}{3}
$$

Case 2.2.3 $d^{\prime}>1$ and $j=0$. Then, $\bar{n}=2^{\beta} d^{\prime}$ and $2 \bar{n}=2^{\beta+1} d^{\prime}$. Hence,

$$
\frac{B_{\bar{n},-1, q}}{n}=\frac{1}{d^{\prime}} \leq \frac{1}{3}
$$


Case 2.2.4 $d^{\prime}>1$ and $j=1$. Then, $\bar{n}=d^{\prime} d_{\alpha_{1}}$. It follows that

$$
\frac{B_{\bar{n},-1, q}}{n} \leq \frac{1}{d^{\prime}} \leq \frac{1}{3}
$$

Case 2.2.5 $j=2$. Then, $\bar{n}=d^{\prime} d_{\alpha_{1}} d_{\alpha_{2}}$. We have

$$
\frac{B_{\bar{n},-1, q}}{n} \leq \frac{1}{d^{\prime} d_{\alpha_{2}}} \leq \frac{1}{d_{\alpha_{2}}} \leq \frac{1}{3} .
$$

Case 2.2.6 $j \geq 3$. Then, $\bar{n}=d^{\prime} d_{\alpha_{1}} \ldots d_{\alpha_{j}}$. Hence,

$$
\frac{B_{\bar{n},-1, q}}{n} \leq \frac{1}{d^{\prime} d_{\alpha_{2}} \ldots d_{\alpha_{j}}} \leq \frac{1}{d_{\alpha_{j}}} \leq \frac{1}{3} .
$$

Altogether, we have $B_{\bar{n},-1, q} \leq \frac{2 n}{3}$, and, hence,

$$
E(n,-1, q)=\frac{\bar{n}-B_{\bar{n},-1, q}}{4} \geq \frac{n-\frac{2 n}{3}}{4}=\frac{n}{12} .
$$

From Theorem 3 and Corollary 4, we can conclude that the average dimension of the Hull of negacyclic codes of length $n=p^{v} \bar{n}$ over $\mathbb{F}_{q}$ is zero if and only if $\beta+1 \leq \gamma, v=0$, and $2 \bar{n} \in N_{q}$. For the other cases, the average dimension of the Hull of negacyclic codes of length $n=p^{v} \bar{n}$ over $\mathbb{F}_{q}$ is bounded by $\frac{n}{12}$ and $\frac{n}{3}$. In these cases, $E(n,-1, q)$ grows at the same rate as the length $n$ of the codes as $n$ tends to $\infty$.

\section{Conclusions}

Due to their wide applications and links with the efficiency of some algorithms in coding theory, properties of hulls of cyclic codes and their generalization in terms of $\lambda$-constacyclic codes have been extensively studied. The average dimension of the Euclidean hull of cyclic codes has been studied in [9]. A complete study of the average dimension of the Hermitian hull of cyclic and constacyclic codes was given in [11,12]. Therefore, the remaining case is the Euclidean hull of negacyclic codes (see [13]). This paper provides a complete study for this problem. The detailed comparison for the results on the Euclidean case is given in Table 1 and the Hermitian case is given in [12].

Table 1. The lower and upper bounds for $E(n, 1, q)$ and $E(n,-1, q)$.

\begin{tabular}{ccccc}
\hline$\lambda$ & $n=p^{v} \bar{n}$ & Lower Bounds & Upper Bounds & Remarks \\
\hline \multirow{2}{*}{1} & $n \in N_{q}$ & 0 & 0 & Theorem 25 of [9] \\
& $n \notin N_{q}$ & $\frac{n}{12}$ & $\frac{n}{3}$ & \\
\hline & $\beta+1>\gamma$ & $\frac{n}{4}$ & $\frac{n}{3}$ & Corollary 4 \\
\cline { 2 - 5 }-1 & $\beta+1 \leq \gamma, v=0$, and $2 \bar{n} \in N_{q}$ & 0 & 0 & \\
& $\beta+1 \leq \gamma, v>0$, and $2 \bar{n} \in N_{q}$ & $\frac{n}{6}$ & $\frac{n}{4}$ & Theorem 3 \\
& $\beta+1 \leq \gamma$ and $2 \bar{n} \notin N_{q}$ & $\frac{n}{12}$ & $\frac{n}{3}$ & \\
\hline
\end{tabular}

Author Contributions: E.S. gave the initial concept and established the results in Section 4. S.J. stated and proved the results in Section 3. S.J. and E.S. wrote the paper.

Acknowledgments: This research was supported by the Thailand Research Fund and the Office of Higher Education Commission of Thailand under Research Grant MRG6080054.

Conflicts of Interest: The authors declare no conflict of interest. 


\section{References}

1. Assmus, E.F.; Key, J.D. Affine and projective planes. Discrete Math. 1990, 83, 161-187. [CrossRef]

2. Leon, J. Computing automorphism groups of error-correcting codes. IEEE Trans. Inf. Theory 1982, 3, 496-511. [CrossRef]

3. Leon, J. Permutation group algorithms based on partition, I: theory and algorithms. J. Symbolic Comput. 1991, 12, 533-583. [CrossRef]

4. Sendrier, N. Finding the permutation between equivalent binary code. In Proceedings of the IEEE International Symposium on Information Theory, Ulm, Germany, 29 June-4 July 1997.

5. Sendrier, N. Finding the permutation between equivalent codes: The support splitting algorithm. IEEE Trans. Inf. Theory 2000, 46, 1193-1203. [CrossRef]

6. Sendrier, N.; Skersys, G. On the computation of the automorphism group of a linear code. In Proceedings of the 2001 IEEE International Symposium on Information Theory, Washington, DC, USA, 29 June 2001.

7. Guenda, K.; Jitman, S.; Gulliver, T.A. Constructions of good entanglement-assisted quantum error correcting codes. Des. Codes Cryptogr. 2018, 86, 121-136. [CrossRef]

8. Sendrier, N. On the dimension of the hull. SIAM J. Appl. Math. 1997, 10, 282-293. [CrossRef]

9. Skersys, G. The average dimension of the hull of cyclic codes. Discrete Appl. Math. 2003, 128, $275-292$. [CrossRef]

10. Sangwisut, E.; Jitman, S.; Ling, S.; Udomkavanich, P. Hulls of cyclic and negacyclic codes over finite fields. Finite Fields Appl. 2015, 33, 232-257. [CrossRef]

11. Jitman, S.; Sangwisut, E. The average dimension of the Hermitian hull of cyclic codes over finite fields of square order. AIP Conf. Proc. 2016 1775, 030026.

12. Jitman, S.; Sangwisut, E. The average dimension of the Hermitian hull of constacyclic codes over finite fields. arXiv 2017, arXiv:1702.00275.

13. Yang, Y.; Cai, W. On self-dual constacyclic codes over finite fields. Des. Codes Cryptogr. 2015, 74, 355-364. [CrossRef]

(C) 2018 by the authors. Licensee MDPI, Basel, Switzerland. This article is an open access article distributed under the terms and conditions of the Creative Commons Attribution (CC BY) license (http:/ / creativecommons.org/licenses/by/4.0/). 